\title{
Mathematical Modeling on the Control of Hunting Problems
}

\author{
Redemtus Heru Tjahjana ${ }^{1 *}$ and Dhimas Mahardika² \\ ${ }^{1-2}$ Department of Mathematics, Faculty of Science and Mathematics, Diponegoro University \\ Jln. Prof. Sudarto, Tembalang, Semarang, Jawa Tengah 50275, Indonesia \\ 1heru.math.undip@gmail.com; ${ }^{2}$ kerajaansanggung@gmail.com
}

Received: $4^{\text {th }}$ May 2020/ Revised: $13^{\text {th }}$ August 2020/ Accepted: $18^{\text {th }}$ August 2020

How to Cite: Tjahjana, R. H., \& Mahardika, D. (2021). Mathematical Modeling on the Control of Hunting Problems. ComTech: Computer, Mathematics and Engineering Applications, 12(1), 1-8. https://doi.org/10.21512/comtech.v12i1.6424

\begin{abstract}
Modeling a natural phenomenon or the action mechanism of a tool is often done in science and technology. Observations through computer simulations cost less relatively. In the research, a bullet control model moving towards the target was explored. The research aimed to try to simulate the trajectory of the bullet that could be controlled in hunting. To model a controlled bullet, the Dubins model was used. Then, the used approach was control theory. The optimal trajectory and control for bullets were designed using the Pontryagin Maximum Principle. The results show that with this principle and the dynamic system of the bullet, a system of differential equations and adjoining is obtained. The fundamental problem arises because the bullet dynamics model in the form of a differential equation system has initial and final requirements. However, the adjoint matching system has no conditions at all. This problem is solved by using numerical methods. In addition, the research proves the convergence of the calculation results with the required results. The track simulation results are also reported at the end of the research to ensure a successful control design. From the simulation results, the presented method with its convergence has successfully solved the problem of bullet control.
\end{abstract}

Keywords: mathematical modeling, bullet control model, hunting problems

\section{INTRODUCTION}

Modeling a natural phenomenon or the action mechanism of a tool is often done in science and technology. The research describes the modeling of a bullet that can be controlled to shoot at a target. A bullet that can move and turn at a fixed height is chosen to be the research topic. It aims to try to simulate the trajectory of a bullet that can be controlled in hunting.
Direct observation of moving bullets is relatively difficult to do and dangerous. However, it is not difficult and dangerous if the observations are made on simulated trajectories of controlled bullet movements. Observations through computer simulations cost less relatively than firing live bullet. In other words, observation by simulation is cheaper and safer than observation by an actual bullet. Observation with simulation is also always done before the controlled bullet is made and fired. In the research, to model a controlled bullet, the Dubins model is used.

Until this year, many researchers have studied the Dubins model. This fact indicates that Dubins model is still an actual object to research. Chen (2020) analyzed Dubins paths in a circle path. Next, Drchal, Faigl, and Váňa (2020) studied windowing surrogate model with Dubins model. Meanwhile, Chen and Shima (2019) studied the shortest Dubins paths through three points. At the same year, Asfihani, Subchan, Rosyid, and Sulisetyono (2019) exposed Dubins path tracking controller. Next, Ding, Xin, and Chen (2019) explored the curvature-constrained path for Dubins vehicle. Furthermore, Parlangeli (2019) explored the shortest paths for Dubins vehicles. After that, Patsko and Fedotov (2018) wrote attainability set instantly for one-side turning Dubins car. Meanwhile, Yao, Qi, Zhao, and Wan (2017) studied bounded curvature path planning with expected length for Dubins vehicle. Next, Váňa, Faigl, Sláma, and Pěnička (2017) researched data collection planning with Dubins model. Several years ago, González, Monje, Moreno, and Balaguer (2016) researched the square method for Unmanned Aerial Vehicle (UAV) mission planning with consideration of Dubins model constraints. In the same year, Marino, Salaris, and Pallottino (2016) researched the controllability of the Dubins model. Before that, Meyer, Isaiah, and Shima (2015) used the Dubins model to intercept a moving target in minimum time. 
Next, the general comparison among the mentioned research with the research can be reported as follows. The difference between the research and Chen (2020) is in the path. Chen (2020) mentioned the circle path. However, the research does not consider the circle path. Next, Drchal et al. (2020) mentioned windowing surrogate model, but the research does not use it. Then, the research is different from Chen and Shima (2019) in the path design. They considered three points that had to be met in the Dubins vehicle. However, the research considers two points as initial position and final position and one orientation as initial orientation and other orientation as final orientation. Meanwhile, Asfihani et al. (2019) exposed path tracking. The research does not expose tracking. Compared to Ding et al. (2019), the research does not use curvature-constrained for optimal path design.

Then, Parlangeli (2019) explored the shortest trajectory via points. Meanwhile, the research exposes optimal trajectory design that satisfies initial and final conditions, including points and orientation. Moreover, Patsko and Fedotov (2018) utilized an attainability set for path design in their research. However, the research uses Pontryagin Maximum Principle and optimal control approach. Similar to Ding et al. (2019), Yao et al. (2017) also utilized curvature-constrained for path design. The research does not consider curvatureconstrained for optimal path design. Then, Váňa et al. (2017) utilized data collection planning. The research does not use it. Next, González et al. (2016) explored the square method for path planning design for the Dubins model for UAVs mission. On the other hand, the research does not use the square method in its research process. Compared to Meyer et al. (2015), who used different cost functional $J$ to minimize the time, the research minimizes the cost controls. The Pontryagin Maximum Principle is used in another research. Ohsawa (2015) explored contact geometry using it.

The novelty of the research is the mathematical convergence proof of the used numerical method to solve the problem of the absence of conditions or values for adjoint variables. Another novelty is the utilization of the Dubins model to describe the dynamics of the motion of the bullet in the hunting problem. The Dubins model is a simple non-linear mathematical model with multi-input and output.

\section{METHODS}

The bullet dynamical model that moves with a fixed height can be presented as follows.

$$
\begin{aligned}
& \dot{x}_{1}=\left(\sin x_{3}\right) u_{1} \\
& \dot{x}_{2}=\left(\cos x_{3}\right) u_{1} \\
& \dot{x}_{3}=u_{2} .
\end{aligned}
$$

There are several reasons why Equation (1) is selected in the research. First, it is simple, but it can describe the moving bullet in fixed height. Second, it is interesting because if linearization at the equilibrium point is applied, the model becomes uncontrollable. However, in the non-linear form, the model is controllable. Third, it contains two essential controls or inputs. The first control is for the speed of the bullet, and the second one is for direction control.

Considering the bullet dynamical model in Equation (1), $\left(x_{1}, x_{2}\right)$ is the position of the bullet and $x_{3}$ is orientation of the bullet. The controls for the bullet are $u_{1}$ and $u_{2}$. The first control is $u_{1}$. This control influences the velocity of the bullet. The second one, $u_{2}$, controls the direction of the bullet. For the detail, for controllability analysis of the bullet dynamical model in Equation (1), it can refer to Marino et al. (2016). Shortly, the bullet dynamical model in Equation (1) can be presented in the general form of non-linear control system Affine, with

$$
\mathbf{x}=\left[\begin{array}{l}
x_{1} \\
x_{2} \\
x_{3}
\end{array}\right], \mathbf{f}(\mathbf{x})=\mathbf{0}, \mathbf{g}_{1}=\left[\begin{array}{c}
\sin x_{3} \\
\cos x_{3} \\
0
\end{array}\right] \text {, and } \mathbf{g}_{2}=\left[\begin{array}{l}
0 \\
0 \\
1
\end{array}\right]
$$

Then, the following process to analyze the controllability is the determination of the controllability matrix. In this process, the Lie Algebra or operation of the Lie bracket is needed. After the controllability matrix is obtained and computation of its rank is performed, the controllability matrix has the third rank. From this fact, it can be concluded that the bullet dynamical model in Equation (1) is locally accessible. Since it is $\mathrm{f}(\mathrm{x})=0$, the bullet dynamical model (1) is controllable. Controllability analysis of the bullet dynamical model in Equation (1) must be performed in non-linear analysis. If the analysis uses the linearization form in Equation (1), the result is not controllable. Controllability analysis is a very important thing. If the model is not controllable, it is impossible to design its control.

Then, the bullet starts moving from an initial position and orientation to the target position and orientation. The equation can be seen as follows.

$$
\left(x_{1}(0), x_{2}(0), x_{3}(0)\right) \text { to }\left(x_{1}(T), x_{2}(T), x_{3}(T)\right) .
$$

Seeing the initial and boundary conditions in Equation (2), the abscissa and the ordinate of the bullet position are denoted by $x_{1}$ and $x_{2}$, respectively. The orientation of the bullet is denoted by $x_{3}$ Then, 0 is the initial time, and $T$ is the final time. With optimal control method, the minimized cost functional model can be formulated:

$$
J=\frac{1}{2} \int_{0}^{T}\left(\delta u_{1}^{2}+\delta u_{2}^{2}\right) d t
$$

In the cost functional model in Equation (3), $\delta$ is a positive constant denoted by the weight of each control. In control theory, the weight of the controls can be equal or different. In the research, the weight of $u_{1}$ and $u_{2}$ are assumed equal to each other and the value of $\delta$ is one. 
The existence analysis of the optimal control problem will also be discussed. The key of existence analysis is based on convex function. Considering the cost functional model in Equation (3), $\delta u_{1}{ }^{2}$ and $\delta u_{2}{ }^{2}$ are convex functions, respectively. Since the sum of the convex function is also a convex function, the cost functional model in Equation (3) is a convex function. Since the cost functional model in Equation (3) is a convex function, the optimal control problem exists. Until now, the existence of optimal control problem in the research is guaranteed to exist mathematically. If existence is not guaranteed, optimal control may not be available when it is sought through any methods.

By considering the two controls of the bullet, many trajectories can be taken by the bullet to move from the initial position to the target position. However, with the optimal control design, the optimal trajectory can also be determined for the bullet. The design of optimal control for bullet uses the Pontryagin Maximum Principle. From the bullet model dynamics in Equation (1) and cost functional model in Equation (3), the Hamiltonian function $(H)$ can be determined. The Hamiltonian function connects the bullet dynamical model in Equation (1) and the cost functional model that must be minimized in Equation (3). Then, the Hamiltonian system is determined through the partial derivative of Hamiltonian function following state variables: $x_{1}, x_{2}$, and $x_{3}$ respectively and partial derivative of Hamiltonian function regarding adjoint variables: $p_{1}, p_{2}$, and $p_{3}$, respectively. Next, through the utilization of the Pontryagin Maximum Principle, the research can derive Hamiltonian function partially about the controls of $u_{1}$ and $u_{2}$ respectively and equate it with 0 .

\section{RESULTS AND DISCUSSIONS}

From the Hamiltonian system and the Pontryagin Maximum Principle, the controls can be derived for the bullet. The controls for the bullet can be described in Theorem 1 as follows.

\section{Theorem 1}

The controls for the bullet are given as follows.

$$
\begin{aligned}
& u_{1}=\frac{\left(p_{1} \sin x_{3}+p_{2} \cos x_{3}\right)}{\delta} \\
& u_{2}=\frac{p_{3}}{\delta}
\end{aligned}
$$

\section{Proof:}

From Equation (1) and cost functional in Equation (3), the Hamiltonian function can be presented.

$$
\begin{aligned}
H & =p_{1}\left(u_{1} \sin x_{3}\right)+p_{2}\left(u_{1} \cos x_{3}\right) \\
& +p_{3} u_{2}+\frac{1}{2} p_{0} \delta u_{1}^{2}+\frac{1}{2} p_{0} \delta u_{2}^{2} .
\end{aligned}
$$

Now, the model dynamical system of the bullet in Equation (1) and cost functional model in Equation
(3) are put together in the Hamiltonian function. Then, the Hamiltonian system can be obtained as follows.

$$
\begin{aligned}
& \frac{\partial H}{\partial p_{1}}=\dot{x}_{1}=u_{1} \sin x_{3} \\
& \frac{\partial H}{\partial p_{2}}=\dot{x}_{2}=u_{1} \cos x_{3} \\
& \frac{\partial H}{\partial p_{3}}=\dot{x}_{3}=u_{2} \\
& \frac{\partial H}{\partial x_{1}}=-\dot{p}_{1}=0 \\
& \frac{\partial H}{\partial x_{2}}=-\dot{p}_{2}=0 \\
& \frac{\partial H}{\partial x_{3}}=-\dot{p}_{3}=\left(p_{1} u_{1} \cos x_{3}\right)-\left(p_{2} u_{1} \sin x_{3}\right) .
\end{aligned}
$$

Meanwhile, the stationary condition from Pontryagin Maximum Principle gives the results as follows.

$$
\begin{aligned}
& \frac{\partial H}{\partial u_{1}}=0=p_{1} \sin x_{3}+p_{2} \cos x_{3}+p_{0} \delta u_{1} \\
& \frac{\partial H}{\partial u_{2}}=0=p_{3}+p_{0} \delta u_{2} .
\end{aligned}
$$

With simple manipulation, the research takes value $p_{0}=-1$ and considers the differential system in Equation (7). The controls for bullet can be presented as follows.

$$
\begin{aligned}
& u_{1}=\frac{\left(p_{1} \sin x_{3}+p_{2} \cos x_{3}\right)}{\delta} \\
& u_{2}=\frac{p_{3}}{\delta} .
\end{aligned}
$$

From the system of Equation (8), the analytic controls or formula of controls bullet are obtained, but the problem keeps remaining. The main problem is the bullet must move to meet the final conditions of $\left(x_{1}(T), x_{2}(T), x_{3}(T)\right)$ which is presented in Equation (2). Therefore, from Equations (6) and (7), the control of Equation (8) is obtained. Then, a system of differential equations that must be solved numerically is as follows.

$$
\begin{aligned}
& \dot{x}_{1}=\frac{1}{\delta}\left(p_{1} \sin ^{2} x_{3}+p_{2} \sin x_{3} \cos x_{3}\right) \\
& \dot{x}_{2}=\frac{1}{\delta}\left(p_{1} \sin x_{3} \cos x_{3}+p_{2} \cos ^{2} x_{3}\right) \\
& \dot{x}_{3}=\frac{1}{\delta} P_{3} \\
& -\dot{p}_{1}=0 \\
& -\dot{p}_{2}=0 \\
& -\dot{p}_{3}=p_{1} \dot{x}_{2}+p_{2} \dot{x}_{1} .
\end{aligned}
$$

In solving the differential equation system in Equation (9) numerically, the problem arises. The 
state variables that exist in the ascending bullet system are $x_{1}, x_{2}$ and $x_{3}$ They have initial and final values. However, the adjoint variables $\left(p_{1}, p_{2}\right.$, and $\left.p_{3}\right)$ do not have specific requirements. If variables of $p_{1}, p_{2}$ and $p_{3}$ use any values, the bullet cannot reach the final target, final position, or final orientation. If the trial and error method is used to substitute the values for the adjoint variable, the final target of the bullet will be challenging to reach the target. This problem is solved by a numerical method which is explained in Theorem 2 . In this theorem, the convergence of the computation results is also proven. The detail of Theorem 2 and its proof can be seen as follows.

\section{Theorem 2}

Through the numerical method, which is employed, the calculated values will converge to the required final values.

\section{Proof:}

The system of differential equations in Equation (9) is solved by considering it as the initial value problem. First, the initial conditions for $p_{1}, p_{2}$, and $p_{3}$ are given arbitrary values, namely $p_{1}(0)=L_{10}, p_{2}(0)=L_{20}$, and $p_{3}(0)=L_{30}$. Next, the error tolerance is set. Then, from the conditions for $\mathrm{x}_{1}(0), \mathrm{x}_{2}(0)$ and $\mathrm{x}_{3}(0)$ and the system in Equation (8), the first iteration is done. From this process, $\left(x_{1}(T)_{1}, x_{2}(T)_{1}, x_{3}(T)_{3}\right)=\mathbf{X}(\mathbf{T})_{1}$ and $\left\|\mathbf{X}(\mathbf{T})_{1}-\mathbf{X}(\mathbf{T})\right\|$ are obtained. If the value of $\left\|\mathbf{X}(\mathbf{T})_{1}-\mathbf{X}(\mathbf{T})\right\|$ is less than or as same as the error tolerance value, the calculation process stops. If it is not, the initial conditions for the adjoint variables of $p_{1}, p_{2}$, and $p_{3}$ are corrected with following the calculations:

$$
\begin{aligned}
& L_{11}=L_{10}-\alpha \frac{\frac{\partial F}{\partial L_{1}}}{\left\|\frac{\partial F}{\partial L_{1}}, \frac{\partial F}{\partial L_{2}}, \frac{\partial F}{\partial L_{3}}\right\|} \\
& L_{21}=L_{20}-\alpha \frac{\frac{\partial F}{\partial L_{2}}}{\left\|\frac{\partial F}{\partial L_{1}}, \frac{\partial F}{\partial L_{2}}, \frac{\partial F}{\partial L_{3}}\right\|} \\
& L_{31}=L_{30}-\alpha \frac{\frac{\partial F}{\partial L_{3}}}{\left\|\frac{\partial F}{\partial L_{1}}, \frac{\partial F}{\partial L_{2}}, \frac{\partial F}{\partial L_{3}}\right\|}
\end{aligned}
$$

with

$$
\begin{aligned}
& \frac{\partial F}{\partial L_{1}} \approx \frac{F\left(L_{10}+\omega, L_{20}, L_{30}\right)-F\left(L_{10}, L_{20}, L_{30}\right)}{\omega} \frac{\partial F}{\partial L_{2}} \approx \frac{F\left(L_{10}, L_{20}+\omega, L_{30}\right)-F\left(L_{10}, L_{20}, L_{30}\right)}{\omega} \\
& \frac{\partial F}{\partial L_{3}} \approx \frac{F\left(L_{10}, L_{20}, L_{30}+\omega\right)-F\left(L_{10}, L_{20}, L_{30}\right)}{\omega}
\end{aligned}
$$

Here, $\alpha$ and $\omega$ are positive constant and $F=\left\|\mathrm{x}(T)_{1}-\mathrm{x}(T)\right\|^{2}$. Next, the second iteration involves the system of differential equations in Equation (9) with the new initial conditions for $p_{1}, p_{2}$, and $p_{3}$ as follows.

$$
\begin{aligned}
& x_{1}(0)=x_{10} \\
& x_{2}(0)=x_{20} \\
& x_{3}(0)=x_{30} \\
& p_{1}(0)=L_{11} \\
& p_{2}(0)=L_{21} \\
& p_{3}(0)=L_{31}
\end{aligned}
$$$$
\text { obtained }\left(x_{1}(T)_{2}, x_{2}(T)_{2}, x_{3}(T)_{2}\right)=\mathbf{X}(\mathbf{T})_{2} \text { and }
$$$$
\left\|\mathbf{X}(\mathbf{T})_{2}-\mathbf{X}(\mathbf{T})\right\| \text {. }
$$

Next, the research compares the value of $\left\|\mathbf{X}(\mathbf{T})_{2}-\mathbf{X}(\mathbf{T})\right\|$ and the error tolerance value. If the value of $\left\|\mathbf{X}(\mathbf{T})_{2}-\mathbf{X}(\mathbf{T})\right\|$ is greater than the error tolerance, it is similar to correction for the initial conditions for $p_{1}$, $p_{2}$ and $p_{3}$ in the second iteration. The third iteration is done with the new initial conditions $p_{1}, p_{2}$, and $p_{3}$ as follows.

$$
\begin{aligned}
& x_{1}(0)=x_{10} \\
& x_{2}(0)=x_{20} \\
& x_{3}(0)=x_{30} \\
& p_{1}(0)=L_{12} \\
& p_{2}(0)=L_{22} \\
& p_{3}(0)=L_{32} \\
& \text { obtained }\left(x_{1}(T)_{3}, x_{2}(T)_{3}, x_{3}(T)_{3}\right)=\mathbf{X}(\mathbf{T})_{3} \text { and } \\
& \left\|\mathbf{X}(\mathbf{T})_{3}-\mathbf{X}(\mathbf{T})\right\| .
\end{aligned}
$$

Similar to after the second iteration, verification of $\left\|\mathbf{X}(\mathbf{T})_{3}-\mathbf{X}(\mathbf{T})\right\|$ and the error tolerance value are performed. If the value of $\left\|\mathbf{X}(\mathbf{T})_{3}-\mathbf{X}(\mathbf{T})\right\|$ is greater than the error tolerance, the iteration continues to the next iteration. The iteration is repeated until the $(n-1)$ th iteration. Next, if the value of $\left\|\mathbf{X}(\mathbf{T})_{n-1}-\mathbf{X}(\mathbf{T})\right\|$ is still greater than the error tolerance, the $n$-th iteration is done with the new initial conditions for $p_{1}, p_{2}$, and $p_{3}$ as follows.

$$
\begin{aligned}
& x_{1}(0)=x_{10} \\
& x_{2}(0)=x_{20} \\
& x_{3}(0)=x_{30} \\
& p_{1}(0)=L_{1(n-1)} \\
& p_{2}(0)=L_{2(n-1)} \\
& p_{3}(0)=L_{3(n-1} \\
& \text { obtained }\left(x_{1}(T)_{n}, x_{2}(T)_{n}, x_{3}(T)_{n}\right)=\mathbf{X}(\mathbf{T})_{n} \text { and } \\
& \left\|\mathbf{X}(\mathbf{T})_{n}-\mathbf{X}(\mathbf{T})\right\| .
\end{aligned}
$$


Until this step, it obtains the number sequence of $\left\|\mathbf{X}(\mathbf{T})_{1}-\mathbf{X}(\mathbf{T})\right\|,\left\|\mathbf{X}(\mathbf{T})_{2}-\mathbf{X}(\mathbf{T})\right\|,\left\|\mathbf{X}(\mathbf{T})_{3}-\mathbf{X}(\mathbf{T})\right\|,\left\|\mathbf{X}(\mathbf{T})_{4}-\mathbf{X}(\mathbf{T})\right\|$ $, \ldots,\left\|\mathbf{X}(\mathbf{T})_{n}-\mathbf{X}(\mathbf{T})\right\|$.

Next, by mentioning the following equations:

$$
\begin{gathered}
\left\|\mathbf{X}(\mathbf{T})_{1}-\mathbf{X}(\mathbf{T})\right\|=M_{1} \\
\left\|\mathbf{X}(\mathbf{T})_{2}-\mathbf{X}(\mathbf{T})\right\|=M_{2} \\
\vdots \\
\left\|\mathbf{X}(\mathbf{T})_{n}-\mathbf{X}(\mathbf{T})\right\|=M_{n},
\end{gathered}
$$

The detail of mathematical proof that the sequence of $M_{1}, M_{2}, M_{3}, \ldots$ will converge to 0 . The iterations of the calculated value from the final value of the state variable will converge to the final value of the required state variable given as follows. The research takes a metric of $d(.$, .) in R. Furthermore, it is assumed that can be taken a continuous function of $\varphi$ with $\varphi:[0, \mathrm{~T}] \epsilon \mathrm{R}$, and $\varphi\left(t_{1}\right)=\left\|X(T)_{t_{1}}-\mathrm{X}(T)\right\|$, for each $t_{1} \in[0, T]$. Because the function of $\varphi$ is continuous, so for each of $\varepsilon>0$, there exists $\rho>0$, such that $\left|t_{1}-t_{2}\right|<\rho \Rightarrow d\left(\varphi\left(t_{1}\right), \varphi\left(t_{2}\right)\right)<\varepsilon$.

$$
\begin{array}{ccc}
\text { Then, the } & \text { result } & \text { obtains } \\
d\left(\left\|X(T)_{t_{1}}-X(T)\right\|,\left\|X(T)_{t_{2}}-X(T)\right\|\right)<\varepsilon . &
\end{array}
$$

There is $d\left(M_{t_{1}}, M_{t_{2}}\right)<\varepsilon$, and it is concluded that the sequence $\left\{\mathrm{M}_{\mathrm{i}}\right\}$ converges to 0 . It means calculated values will converge to the required final value.

Theorem 2 has an important contribution to the research. With numerical methods that have been mathematically proven, the convergence in Theorem 2 is used to solve problems. To ensure that the problemsolving method can be used, several simulations are conducted. Before the simulations are conducted, simulation scenarios are needed to describe how the simulation will be carried out.

There are six simulations in the research. For each simulation, the origin coordinates or initial positions and orientations of the bullet are $(0,0,0)$. Simulation 1 is that the bullet moves from $(0,0,0)$ to the final condition with fixed abscissa and orientation, but the ordinate is changed. The aim of simulation 1 is to verify whether the method can work to control the bullet reach the final conditions with changed ordinate.

Similar to simulation 1 , in the scenario of simulation 2 , the bullet moves from the origin $(0,0,0)$ to final conditions that the ordinate and orientation are changed, and the abscissa is still fixed. Simulation 2 checks whether the method can control the bullet in reaching the final target with the changed ordinate and the orientation. Next, the scenario of simulation 3 is described as follows. The bullet moves from the origin $(0,0,0)$ to the target with the fixed ordinate and orientation and changed abscissa. Simulation 3 shows that the numerical method can work properly if the ordinate and orientation for the target are still fixed, but the abscissa is changed.

Meanwhile, in simulation 4, the bullet moves from the origin $(0,0,0)$ to the final destination. The abscissa and the orientation are changed, but the ordinate is as same as the initial ordinate. Similar to the aim of simulation 3 , simulation 4 is used to ensure the method can reach the final target with the changed abscissa and the orientation and fixed ordinate. Furthermore, in simulation 5, the bullet moves from $(0,0,0)$ to the target with the changed abscissa and the ordinate. However, the orientation is fixed. Simulation 5 shows whether the method can use to solve the problem with the target. The abscissa and the ordinate are changed, and the orientation is fixed.

In the last scenario, simulation 6 , the bullet moves with the changed abscissa, ordinate, and orientation. It aims to check whether the method can work successfully if the bullet moves from the origin by changing all aspects of the target. The described simulation scenarios are summarized in Table 1. According to simulation scenarios, the details of the initial and final target, including abscissa, ordinate, and orientation for each simulation, are shown in Table 2.

Table 1 The Summary of Simulation Scenarios

\begin{tabular}{cccc}
\hline No & Abscissa & Ordinate & Orientation \\
\hline 1. & fixed & changed & fixed \\
2. & fixed & changed & changed \\
3. & changed & fixed & fixed \\
4. & changed & fixed & changed \\
5. & changed & changed & fixed \\
6. & changed & changed & changed \\
\hline
\end{tabular}

Table 2 Initial and Final Conditions

\begin{tabular}{ccc}
\hline Simulation & $\begin{array}{c}\text { Initial Condition } \\
(\mathrm{m}, \mathrm{m}, \mathrm{rad})\end{array}$ & $\begin{array}{c}\text { Final Condition } \\
(\mathrm{m}, \mathrm{m}, \mathrm{rad})\end{array}$ \\
\hline 1 & $(0,0,0)$ & $(0,15,0)$ \\
2 & $(0,0,0)$ & $(0,15, \mathrm{pi} / 2)$ \\
3 & $(0,0,0)$ & $(50,0,0)$ \\
4 & $(0,0,0)$ & $(15,0, \mathrm{pi} / 4)$ \\
5 & $(0,0,0)$ & $(15,15,0)$ \\
6 & $(0,0,0)$ & $(15,15, \mathrm{pi} / 4)$ \\
\hline
\end{tabular}

In the research, all simulations use 0 as the initial time and 1 as the final time. The unit of time is seconds. The simulation is conducted numerically using MATLAB software. MATLAB is chosen because its performance in numerical simulation is well-known. The simulation results, which contain optimal path or optimal trajectory for each simulation, are reported in Figures 1 to 6.

After the simulation results are reported, the researchers discuss the results. Simulation 1 demonstrates bullet moving from $(0,0,0)$ to $(0,15,0)$, which means simulation successfully done with the changed ordinate and the fixed abscissa and 
orientation. The result of simulation 1 is plotted in Figure 1. In Figure 1, the bullet moves from $(0,0,0)$ to $(0,15,0)$, but the optimal trajectory is not in the vertical line because the orientation of the bullet is considered. At the beginning of the movement, the bullet moves with a changed orientation. At the final time, the bullet orientation is back to 0 , or the final orientation is the same as the initial orientation.

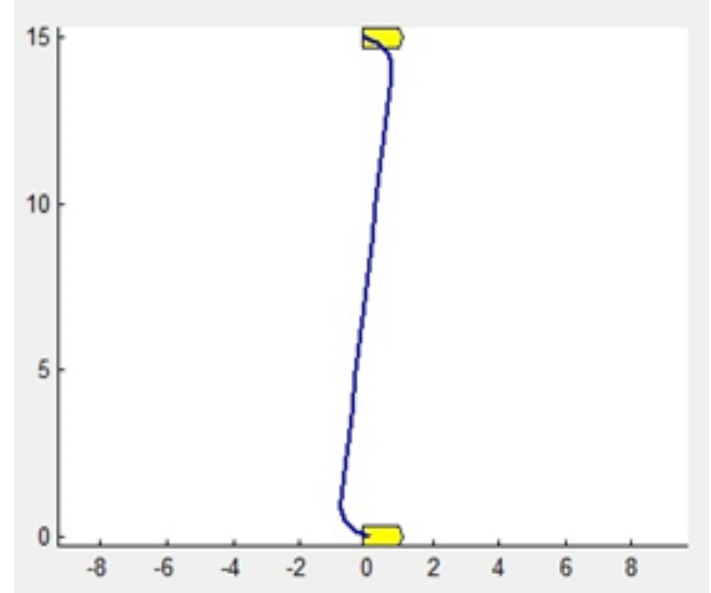

Figure 1 The Optimal Trajectory of Simulation 1

Simulation 2 shows bullet moving from $(0,0,0)$ to $(0,15, \mathrm{pi} / 2)$. The ordinate and orientation are changed, but the abscissa is still fixed. Figure 2 shows the result of simulation 2. In Figure 2, the trajectory is similar to simulation 1 , but the final orientation is $\mathrm{pi} / 2$. It can also be seen that the changed orientation appears until the end of the simulation. At the same time, the ordinate also changes to 15 .

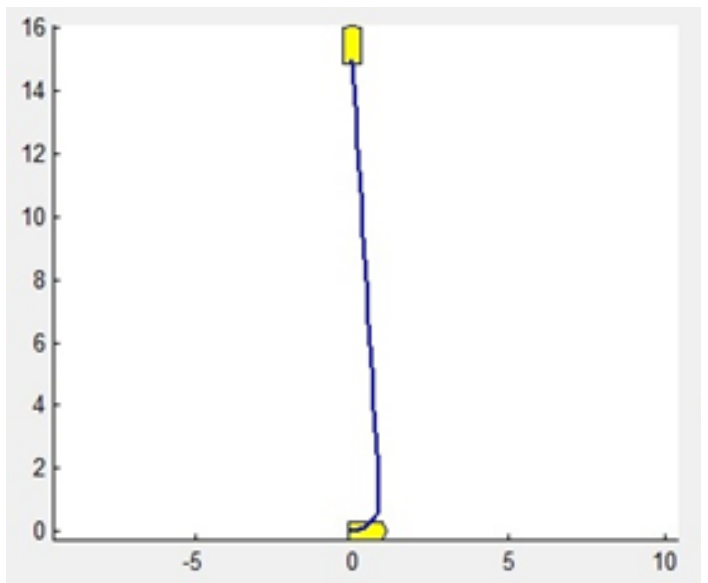

Figure 2 The Optimal Trajectory of Simulation 2

Simulation 3 describes bullet moving from $(0,0,0)$ to $(50,0,0)$. In this simulation, the abscissa is changed, but the ordinate and orientation are fixed. The result of simulation 3 can be seen in Figure 3. The bullet moves horizontally from coordinate $(0,0)$ to coordinate $(50,0)$ without changing the orientation.

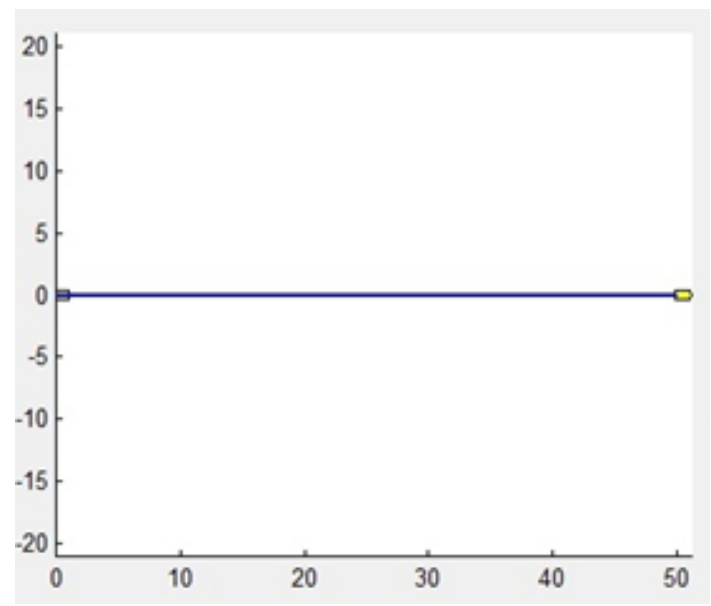

Figure 3 The Optimal Trajectory of Simulation 3

The bullet moving from $(0,0,0)$ to $(15,0, \mathrm{pi} / 4)$ is simulated in simulation 4 . The abscissa and the orientation are changed, but the ordinate is still fixed. The plot of optimal trajectory for simulation 4 is presented in Figure 4. The optimal trajectory of the bullet is similar to the result of simulation 3 . However, the final orientation changes to pi/4 at the final time of the simulation.

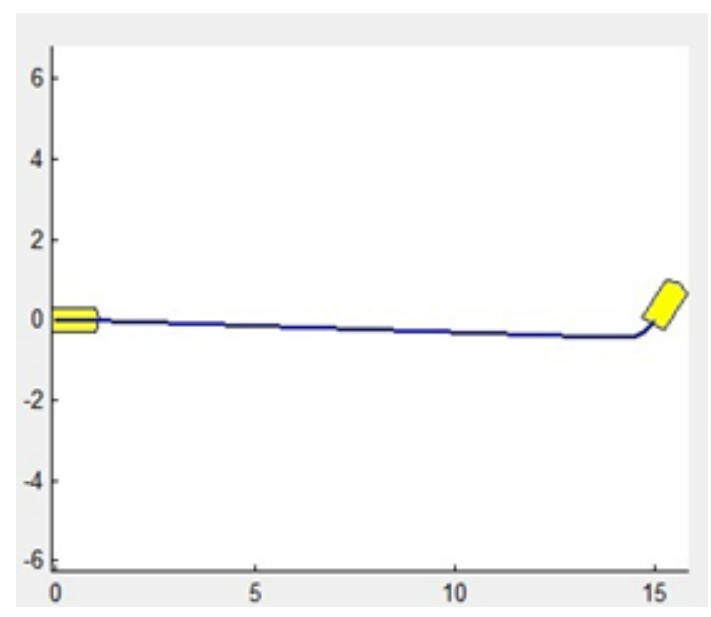

Figure 4 The Optimal Trajectory of Simulation 4

In simulation 5 , the bullet moves from $(0,0,0)$ to $(15,15,0)$. This simulation has a fixed orientation, but the abscissa and the ordinate are changed. The result of simulation 5 is plotted in Figure 5. The optimal trajectory of the bullet can be seen that the orientation of the bullet changes along with the motion. At the final time of simulation, the orientation of the bullet is back to the initial orientation. In other words, the bullet orientation at the final time of simulation is as same as the bullet orientation at the initial time of the simulation. 


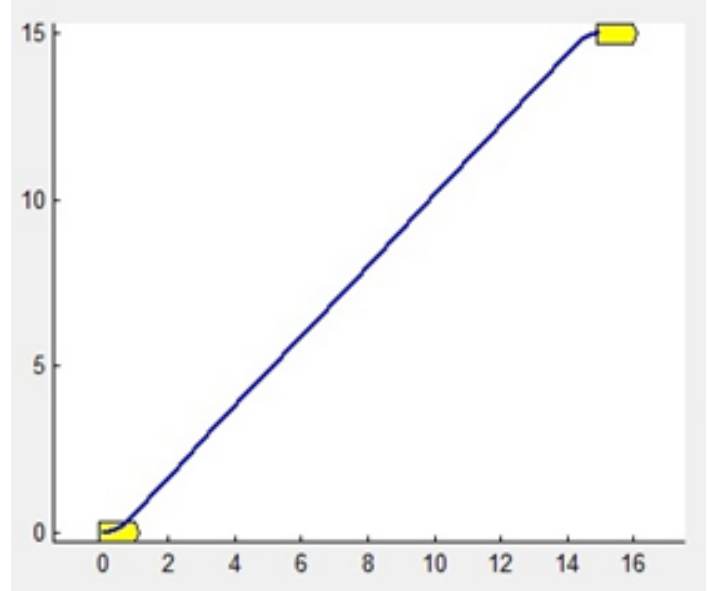

Figure 5 The Optimal Trajectory of Simulation 5

Finally, the bullet moves from $(0,0,0)$ to $(15,15, \mathrm{pi} / 4)$ described in simulation 6 . In the last simulation, all aspects (abscissa, ordinate, and orientation) are different from the initial condition. The result of simulation 6 can be seen in Figure 6 . It can be observed that optimal trajectory simulation 6 is similar to the optimal trajectory of simulation 5 . However, the final orientation of the bullet is different from the initial orientation.

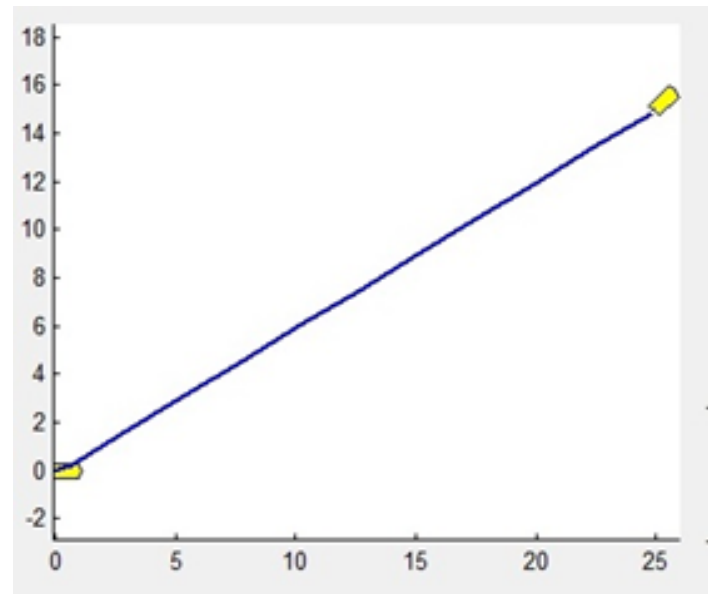

Figure 6 The Optimal Trajectory of Simulation 6

\section{CONCLUSIONS}

Using mathematical modeling (control theory and optimal control theory), the problem of bullet control can be described properly with the bullet dynamical model in Equation (1). The problem is successfully resolved. In solving the problem through the Pontryagin Maximum Principle, another problem arises. There is no requirement for adjoint variables. Then, the solution with numerical methods is used to solve this problem. The convergence of the calculation results with the used numerical method is successfully demonstrated. Then, bullet control simulations are reported. From the simulation results, the presented method with its convergence has successfully solved the problem of bullet control.

The research limitation is no disturbance in the bullet moving. In future research, it is more interesting if the bullet disturbance can be added as a factor that influences the motion of the bullet. An example of a disturbance in a bullet is air friction that blocks the motion of the bullet. Disturbance can be added to the bullet dynamical system equation. Although the friction force of the bullet in the air is relatively small, including the disturbance factor makes the dynamic model of the motion of the bullet closer to actual reality in the field. The side effect of the inclusion of a disturbance factor in the model makes solving the problem relatively more complicated.

\section{ACKNOWLEDGEMENTS}

The authors acknowledge the Faculty of Science and Mathematics (FSM), Diponegoro University (Undip), Semarang, Indonesia, for the funding support under research contract number 1955/ UN7.5.8/PP/2020. The authors would like to thank the suggestions and critics from unknown reviewers for their valuable comments to improve the research quality.

\section{REFERENCES}

Asfihani, T., Subchan, S., M. Rosyid, D. M., \& Sulisetyono, A. (2019). Dubins path tracking controller of USV using model predictive control in sea field. Journal of Engineering and Applied Sciences, 14(20), 7778-7787. https://doi. org/10.36478/jeasci.2019.7778.7787

Chen, Z. (2020). On Dubins paths to a circle. Automatica, 117(July), $\quad 1-8 . \quad$ https://doi.org/10.1016/j. automatica.2020.108996

Chen, Z., \& Shima, T. (2019). Shortest Dubins paths through three points. Automatica, 105(July), 368-375. https:// doi.org/10.1016/j.automatica.2019.04.007

Ding, Y., Xin, B., \& Chen, J. (2019). Curvature-constrained path elongation with expected length for Dubins vehicle. Automatica, 108(October), 1-8. https://doi. org/10.1016/j.automatica.2019.108495

Drchal, J., Faigl, J., \& Váňa, P. (2020). WiSM: Windowing surrogate model for evaluation of curvatureconstrained tours with Dubins vehicle. IEEE Transactions on Cybernetics, 1-10. https://doi. org/10.1109/tcyb.2020.3000465

González, V., Monje, C. A., Moreno, L., \& Balaguer, C. (2016). Fast marching square method for UAVs mission planning with consideration of Dubins model constraints. IFAC-PapersOnLine, 49(17), 164-169. https://doi.org/10.1016/j.ifacol.2016.09.029

Marino, H., Salaris, P., \& Pallottino, L. (2016). Controllability analysis of a pair of 3D Dubins vehicles in formation. Robotics and Autonomous Systems, 83(September), 94-105. https://doi.org/10.1016/j.robot.2016.05.015

Meyer, Y., Isaiah, P., \& Shima, T. (2015). On Dubins 
paths to intercept a moving target. Automatica, 53(March), 256-263. https://doi.org/10.1016/j. automatica.2014.12.039

Ohsawa, T. (2015). Contact geometry of the Pontryagin maximum principle. Automatica, 55, 1-5. https://doi. org/10.1016/j.automatica.2015.02.015

Parlangeli, G. (2019). Shortest paths for Dubins vehicles in presence of via points. IFAC-PapersOnLine, 52(8), 295-300. https://doi.org/10.1016/j. ifacol.2019.08.086

Patsko, V., \& Fedotov, A. A. (2018). Attainability set at instant for one-side turning Dubins car. IFACPapersOnLine, 51(32), 201-206. https://doi. org/10.1016/j.ifacol.2018.11.381

Váňa, P., Faigl, J., Sláma, J., \& Pěnička, R. (2017). Data collection planning with Dubins airplane model and limited travel budget. In 2017 European Conference on Mobile Robots (ECMR) (pp. 1-6). https://doi. org/10.1109/ecmr.2017.8098715

Yao, W., Qi, N., Zhao, J., \& Wan, N. (2017). Bounded curvature path planning with expected length for Dubins vehicle entering target manifold. Robotics and Autonomous Systems, 97(November), 217-229. https://doi.org/10.1016/j.robot.2017.09.003 